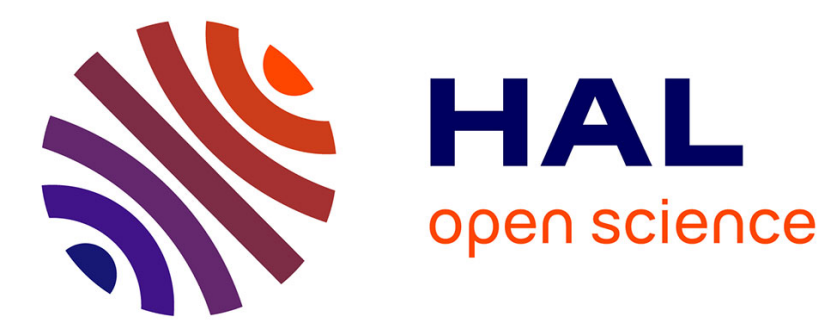

\title{
Control Beliefs and Engagement in Hygienic and Safety Behaviours: The Case of Foodborne illness
}

Dongo Rémi Kouabenan, Robert Ngueutsa Ngueutsa

\section{To cite this version:}

Dongo Rémi Kouabenan, Robert Ngueutsa Ngueutsa. Control Beliefs and Engagement in Hygienic and Safety Behaviours: The Case of Foodborne illness. International Journal of Environmental Health Research, 2015, 26 (4), 10.1080/09603123.2015.1119807 . halshs-01425837

\section{HAL Id: halshs-01425837 \\ https://shs.hal.science/halshs-01425837}

Submitted on 10 Jul 2017

HAL is a multi-disciplinary open access archive for the deposit and dissemination of scientific research documents, whether they are published or not. The documents may come from teaching and research institutions in France or abroad, or from public or private research centers.
L'archive ouverte pluridisciplinaire HAL, est destinée au dépôt et à la diffusion de documents scientifiques de niveau recherche, publiés ou non, émanant des établissements d'enseignement et de recherche français ou étrangers, des laboratoires publics ou privés. 


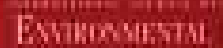

Hixani Risusai

\section{International Journal of Environmental Health Research}

\section{Control beliefs and engagement in hygienic and safety behaviours: the case of foodborne illness}

\section{Dongo Rémi Kouabenan \& Robert Ngueutsa}

To cite this article: Dongo Rémi Kouabenan \& Robert Ngueutsa (2015): Control beliefs and engagement in hygienic and safety behaviours: the case of foodborne illness, International Journal of Environmental Health Research, DOI: 10.1080/09603123.2015.1119807

To link to this article: http://dx.doi.org/10.1080/09603123.2015.1119807

View supplementary material $[\widetilde{T}$

Published online: 30 Dec 2015.

Submit your article to this journal $[\pi$

View related articles ¿

View Crossmark data $ऍ$ 


\title{
Control beliefs and engagement in hygienic and safety behaviours: the case of foodborne illness
}

\author{
Dongo Rémi Kouabenan and Robert Ngueutsa \\ Interuniversity Laboratory of Psychology (LIP/PC2S), University of Grenoble-Alpes, UFR SHS, Grenoble Cedex 09, \\ France
}

\begin{abstract}
Foodborne illness is an ever-growing concern in public health. Studies found that conventional training is not enough to cause employees to apply the hygiene and safety measures. The present study explores control and fatalistic beliefs as potential factors for explaining engagement in preventive actions. Two-hundred and seventeen employees of a fast-food restaurant (75\% of all staff) answered a questionnaire assessing their control beliefs, fatalistic beliefs, risk perception, and engagement in hygienic and safety behaviours. The results validated our hypotheses. Control beliefs were positively related to engagement in hygienic and safety behaviours $(b=0.43$, $p<0.001)$. The inverse relation was observed for fatalistic beliefs $(b=-0.24$, $p<0.001)$. The perceived effectiveness of the prescribed measures seems to be the best predictor of engagement in preventive behaviours, followed by perceived self-efficacy. To increase adherence to preventive measures, it is recommended to enhance staff's self-efficacy and perceived effectiveness of these measures.
\end{abstract}

\section{ARTICLE HISTORY}

Received 25 May 2015

Accepted 9 November 2015

\section{KEYWORDS}

Self-efficacy; effectiveness of protective measures; control beliefs; fatalistic beliefs; risk perception; engagement in protective behaviours

\section{Supplementary_Material.docxIntroduction}

As noted by some authors (Egan et al. 2007; Milton \& Mullan 2012), foodborne illness is an important public health problem that generates substantial costs to individuals and the food industry, and incurs an economic burden for the whole society. According to Milton and Mullan (2012) data reports in the USA estimate that for the year 2010, 48 million consumers were affected, causing 128,000 hospitalizations and 3000 deaths and generating a mean economic cost of approximately 152 billion dollars annually. "Within England and Wales the number of foodborne illness notifications rose steadily from approximately 15,000 cases in the early 1980s to a peak of over 60,000 cases in 1996" (Egan et al. 2007, p. 1180). Chow and Mullan (2010) reported that approximately "5.4 million Australians get sick annually from eating contaminated food” (p. 7575). The numbers of foodborne outbreaks have increase in France from 326 reported outbreaks in 1990 to 1153 outbreaks in 2011 which affected 9674 persons, of whom 668 were hospitalised and 7 died (InVS 2011). These statistics show that the two greatest sources of foodborne illness in France are meals served in homes (37.8\%) and in restaurants (36\%). Foodborne illness impacts the health of the affected consumers and represents a threat for the food and restaurant industries. The death in 2011 of a customer shortly after a meal in a French

CONTACT Dongo Rémi Kouabenan Remi.Kouabenan@upmf-grenoble.fr 
fast-food restaurant caused the restaurant chain to suffer a sales decline of $4.6 \%$, despite the fact that the restaurant's responsibility for the death was not established by the courts.

Given the substantial costs generated by foodborne illness outbreaks, preventing its occurrence has become a critical issue. Human behaviours are very often mentioned as the cause (Clayton \& Griffith 2008; Mullan \& Wong 2009; Yiannas 2009; Chow \& Mullan 2010; Milton \& Mullan 2010; Wright \& Leach 2013). This suggests that modifying these behaviours could greatly contribute to reducing food contamination. Yet, few psychological studies have been conducted to find out what determines these human behaviours in the area of foodborne illness, and there are even fewer studies on fastfood restaurants. The training programmes proposed generally consist of recommending actions like "washing and drying the hands correctly for at least 20 seconds, keeping surfaces and equipment clean, separating raw and cooked food, cooking food thoroughly, keeping food at safe temperatures, and using safe water and raw materials" (World Health Organization, 2006, quoted by Milton \& Mullan 2012 , p. 250). To achieve these goals, training courses are held. However, according to Clayton et al. (2002) standard training in hygiene and safety is not enough to cause employees to apply the prescribed measures. Similarly, Yiannas (2009) considered that "achieving food safety success in this changing environment often requires going beyond traditional training, testing, and inspectional approaches to managing risks. It requires a better understanding of organizational culture and the human dimensions of food safety" (p. 1). Like many other authors (Griffith et al. 2010; Powell et al. 2011; Wright \& Leach 2013), he proposes to change the behaviour of the people involved in food management through the introduction of a food safety culture. As stated by Wright and Leach (2013), "food safety culture is now coming to the fore" (p. 1). According to them, food safety culture is viewed as "how and what the employees in a company or organisation think about food safety as well as the food safety behaviours that they routinely practice and demonstrate" (p. 3). Griffith et al. (2010) defined food safety culture as "the aggregation of the prevailing relatively constant, learned, shared attitudes, values and beliefs contributing to the hygiene behaviours used in a particular food handling environment" (p. 435). For Yiannas (2009), to improve food safety performance, it is necessary to create a behaviour-based food safety management system. To change the behaviour, Clayton et al. (2002) proposed, like others (Slovic et al. 1981; Kouabenan 1998, 2009), taking beliefs and perceptions into account to help improve training and lead employees to be highly committed to engaging in hygienic and safety behaviours.

Taking beliefs into account is becoming a widely-used exploratory approach for gaining insight into health and safety issues, and for defining more effective and longer lasting preventive actions (Weinstein 1993; Kouabenan 1998; Sloan et al. 2009; Bergvik et al. 2012; Kayani et al. 2012). The initial hypothesis in these studies is that beliefs influence risk perceptions and risk-related behaviours (Kouabenan 2006). It would seem that beliefs compensate for the lack of a rational explanation, give meaning to events, help in making inferences about the relationships between events and provide possible ways of counteracting or preventing harmful effects (Kouabenan 2007). Beliefs have played a key role in studies dealing with the processes underlying the adoption of health-related behaviours (Rogers 1983; Ajzen 1985; Becker \& Rosenstock 1987; Weinstein 1988, 1993; Dejoy 1996; Albarracin et al. 2001). The usual assumption in such studies is that attitudes, beliefs and expectations influence people's adoption or continued execution of safe behaviours, generally in an indirect manner by way of their effects on behavioural intentions.

\section{Control beliefs and protective behaviours}

This study focuses on two aspects of control beliefs as potential determinants of employees' engagement in prevention. The first is perceived self-efficacy, operationalized here by the perceived ability to apply hygienic and safety measures; the second is the perceived effectiveness of the rules, operationalized by a measure of the perceived effectiveness of those measures. Studying the impact of restaurant employees' beliefs on their management of food quality and safety has not only proven to be of theoretical importance, but is also critical from the practical standpoint for recovering consumer confidence, both of which are indispensable in keeping customer fidelity and maintaining an 
acceptable level of consumption (Houghton et al. 2006; Graffeo et al. 2009). According to Graffeo et al. (2009), "Food scares have had, in the short term, major socio-economic consequences, eroding consumer confidence and decreasing the willingness to buy potentially risky food" (p. 59). Houghton et al. (2006) noted similarly that "consumer perceptions of food hazards and how the associated risks are managed are likely to be an important determinant of consumer confidence in food safety" (p. 165).

Self-efficacy beliefs refer to perceptions of one's ability to engage in protective actions; response efficacy beliefs refer the extent to which people believe that the prescribed protective actions are effective (See Bandura 1977; Rogers 1983; Ajzen 1985; Bandura 1997; De Zwart et al. 2009). Studies validating models of the adoption of health-related behaviours (Schwarzer 1992; McCaul et al. 1993; Armitage \& Conner 1999; Albarracin et al. 2001) have shown that among several factors (perceived threat, perceived vulnerability, perceived seriousness of a risk, perceived self-efficacy, attitude toward a given behaviour, etc.), perceived self-efficacy appears to have the greatest impact on safety-related behaviours. Riley and Baah-Odoom (2012) noted that high self-efficacy beliefs were associated with a greater number of healthy behaviours. Likewise, Sloan et al. (2009) reported that individuals with diabetes mellitus (DM) who had stronger beliefs about control over life events and a higher subjective estimation of their longevity engaged more in the recommended DM care practices and had better self-assessed DM control and general health. A comparable result was obtained in Bergvik et al's. (2012) study with patients who had undergone a percutaneous coronary intervention or an artery bypass graft. The authors found a strong association between control beliefs and return to work. In Vaughan's (1993) study on chronic exposure to pesticides, the adoption of self-protective behaviour was shown to be highly probable not only for employees who had received information about the risks, but also for those who had high perceived control and believed that the advocated precautions were effective. In a study on interventions aimed at instigating food-safety behaviours, Milton and Mullan (2012) reported that perceived behavioural control was the only variable that predicted the intention to adopt safe food behaviours. Similarly, Luszcynska (2004) demonstrated that when self-efficacy was activated during an intervention, it was an important predictor of behavioural intentions, planning, and change. On the contrary, fatalistic beliefs which reflect a lack of control over events seem to have an opposite effect. According to Claassen et al. (2010), a fatalistic belief is one held by people who are convinced that they have no power to act upon risks, as opposed to people who view themselves as able to change self-attributes and feel they have a great control. For these authors, fatalistic beliefs can negatively affect engagement in risk-reducing behaviour. This aligns with the results of various studies showing that such beliefs lead people to underestimate risks, neglect safety measures, and execute unsafe behaviours (Kouabenan 1998; Peltzer \& Renner 2003; Kayani et al. 2012).

However, the link between control beliefs and commitment to safety behaviour could be affected by the perception of the risk of food contamination. It seems thus useful to examine the link between the perceived risk of food contamination and engagement in hygienic and safety behaviours. Even if the findings on the link between the perceived risk and safety behaviours sometimes differ (Stasson \& Fishbein 1990; Van der Pligt 1996; Brewer et al. 2007), a large number of studies have shown that risk perceptions have an impact on whether individuals adopt protective behaviours and/or stop executing unhealthy ones (Dejoy 1996; Kuttschreuter 2006; Weinstein et al., 2007; Arezes \& Miguel 2008; Kouabenan 2009; Mbaye \& Kouabenan 2013). Having noted the inconsistency of the results on the link between risk perception and protective behaviours, Rimal and Real (2003) wondered whether perceived self-efficacy might act as a moderating variable. They hypothesised that individuals with a high level of perceived risk and high efficacy beliefs "can be expected to engage in more extensive preventive behaviours than those with high perceived risk and low efficacy beliefs and those with low perceived risk (regardless of efficacy beliefs)" (p. 371). They noted that individuals who had a low perception of risk and low efficacy beliefs were less motivated to engage in self-protective behaviours. 


\section{Study aims and Hypotheses}

One of the originalities of this study is that it focuses on control beliefs and the perceived effectiveness of responses as probable determinants of engagement in preventive behaviours. Another particularity is that it targets food hygiene and safety issues in the fast-food restaurant business. To our knowledge, few studies in psychology have been conducted on this subject. Specifically, we examine the link between control beliefs, the perceived effectiveness of hygienic and safety measures, the perception of risks, and engagement in hygienic and safety behaviours among employees of fast-food restaurants.

Here, we look at the effect of control beliefs on fast-food restaurant employees' engagement in hygienic and safety behaviours. We hypothesise that a high perceived ability of employees to apply the preventive measures predicts greater involvement in hygienic and safety behaviours (Hypothesis 1). Based on our observations in restaurants suggesting that employees react differently, depending on whether the work load is heavy or not, we examined the relationship between this perceived ability and engagement in hygienic and safety behaviours in two different work contexts: normal work periods (Hypothesis 1a) and periods of rush (Hypothesis 1b). We then examine the impact of fatalistic beliefs on these behaviours since fatalism reflects a lack of control over events. Accordingly, we hypothesise that participants who have strong fatalistic beliefs will tend to engage less in hygienic and safety behaviours (Hypothesis 2).

Further we examine the impact of the perceived effectiveness of prescribed hygienic and safety measures by looking at employees' perceptions of the effectiveness of the measures. This dimension seems highly important because it not only raises the question of the adequacy and reliability of the measures, but also the issue of workers' confidence in the effectiveness of those measures. A belief in the effectiveness of a measure could be a critical element in the decision to observe it. It seems unlikely that people would engage in measures they deem useless. We therefore hypothesise that employees will engage in hygienic and safety behaviuours if they feel that the measures they are asked to apply are useful, i.e. likely to actually prevent food contamination (Hypothesis 3).

Lastly, we examine the link between the perceived risk of food contamination and engagement in hygienic and safety behaviours, and the relationship between the different variables of the study (perceived self-efficacy, perceived usefulness of hygiene and safety rules, fatalistic beliefs and perceived risk of contamination). We thus hypothesise that employees who perceive a high risk of food contamination will engage and safety behaviours (Hypothesis 4). In line with the work of Rimal and Real (see above), we hypothesise that the impact of control beliefs, fatalistic beliefs and the perceived effectiveness of measures on engagement in hygienic and safety behaviours will be moderated by the perceived risk of food contamination (Hypothesis 5).

\section{Methodology}

\section{Sample}

The study participants were 217 employees from 14 restaurants in a fast-food restaurant chain. All of the employees had the same rank in the company. Their jobs included producing, storing, and serving meals, as well as various cleaning tasks. They worked under a director assisted by three or four managers. The 14 restaurants were drawn at random from among those located in several different French cities. About $75 \%$ of the employees at the participating restaurants agreed to take part in the study.

\section{Materials and measures}

The study was based on a questionnaire with Likert-like response scales. The questionnaire consisted of items assessing the following variables: perceived ability to apply the hygienic and safety measures during periods with a normal workload and during periods of rush, fatalistic beliefs, perceived effectiveness of hygienic and safety measures, perceived risk regarding actions likely to promote food 
contamination, and engagement in hygienic and safety behaviours. Additionally, the questionnaire contained items measuring the participants' training in hygiene and safety, work experience, and demographics (gender, age and education). It was designed for the purposes of the present study, based on semi-directive interviews with 10 employees from three restaurants in the chain ( 7 workers and 3 directors). The questionnaire was pretested on a sample of 32 employees of the same restaurant chain who were not included in the final study sample. This pretest aimed at checking the understanding of the questions by participants and the reliability of the measurement scales. We checked measurement reliability using the Cronbach reliability test. Items weakly correlated with the measurement scales were removed.

\section{Measure of perceived ability to apply hygienic and safety measures (self-efficacy) during normal and rush periods}

The employees' perceived ability to apply hygienic and safety measures was assessed during two work periods: a normal period when the work load was light, and a rush period when the work load was heavy. It was assessed via a list of 32 hygiene-related measures prescribed by the company (based on our interviews with the directors and workers). The measures pertained to body hygiene (e.g. "Always washing one's hands for 3 times 30 seconds after sneezing or blowing one's nose" and "Having short, clean fingernails"), prevention of cross-contamination (e.g. "Do not move materials from one area to another" and "Do not put cardboard boxes on clean surfaces"), prevention of improper operation of installations (e.g. "Always make sure the refrigerator door is completely closed") and ensuring high-quality food (e.g. "Never serve food that was prepared 15 minutes earlier"). The participants were asked to respond in reference to their real job, either during a normal period or during a rush period. Ratings of how difficult it is to apply each measure were given on a Likert-like scale ranging from 1 (very difficult) to 4 (not difficult at all). Rush periods in France occur at lunch time (noon to $2 \mathrm{pm})$ and at dinner time (6 to $9 \mathrm{pm}$ ).

\section{Measure of perceived effectiveness of hygienic and safety measures}

The perceived effectiveness of the hygienic and safety measures was assessed on a scale containing the same items as those used to assess the perceived ability to apply the prescribed measures. The difference was simply that this time, the participants were asked to rate the usefulness of each of the measures for preventing food contamination, on a Likert-like scale ranging from 1 (not useful at all) to 4 (very useful).

\section{Measure of fatalistic beliefs about food contamination}

Fatalistic beliefs were assessed on a six-item scale based on the fatalism scale first proposed by Kouabenan (1998) and adapted to the present study on the basis of the statements made by the employees during the interviews. The items dealt with the possibility or impossibility of preventing food contamination (e.g. "Food contamination is due to bad luck so there's nothing we can do" and "No matter what you do, you can't prevent food contamination, bacteria are everywhere"). The participants rated their extent of agreement or disagreement with each of the statements on a Likert-like scale ranging from 1 (totally disagree) to 4 (totally agree).

\section{Measure of perceived risk of actions likely to promote food contamination}

Perceived risk was assessed using a list of 24 actions mentioned during the interviews as ones likely to promote food contamination. Other actions were taken from the hygiene and safety training document used by the company. All of the actions were ones that the employees might actually carry out during work and that were likely to promote food contamination (e.g. "Sneezing or blowing one's nose" and "Throwing something away in the garbage can after lifting the cover with one's hand"). The participants were asked to rate what they thought was the probability that each of these actions would promote food contamination, on a scale ranging from 1 (very low probability) to 4 (very high probability). 


\section{Measure of engagement in hygienic and safety behaviours}

To assess whether the employees executed the various hygienic and safety behaviours, the questionnaire described various possible daily work situations. Each situation was followed either by an appropriate reaction (eight pro-trait items) or an inappropriate reaction (nine counter-trait items). The participants were asked to refer to their own habitual behaviour on the job and to rate their extent of agreement or disagreement with the statements, on a scale ranging from 1 (totally disagree) to 4 (totally agree). Some examples of inappropriate reactions to situations are "During a rush period, I sometimes don't wash my hands for 3 times 30 seconds when I go from an unclean area to a clean area" and "When there are too many customers waiting, I can't cook the hamburger until it reaches $69^{\circ}$ ". Some examples of appropriate reactions are "When I see that the temperature of a piece of frozen food is above $0^{\circ} \mathrm{I}$ throw it away" and "When I cut my finger, I put on a bandage and a finger guard before returning to work".

\section{Procedure}

Participants answered the questionnaire individually and took an average of $35 \mathrm{~min}$. The main researcher collected the data, assisted by two psychology students trained in questionnaire administration. After two letters of information addressed by the company to the directors of the restaurants selected, meetings were organised with the director of each restaurant. Appointments were then set up with each employee during his/her work hours at times that would not disrupt food service to customers. The employees agreed to participate without compensation from the researchers. However, the time the employee spent in the interview was paid by the organisation that funded the study. Questionnaire administration took place in a quiet area of the restaurant. To begin, the interviewer explained the purpose of the study, described the questionnaire and told each participant that his/her responses would remain anonymous and confidential. The participant filled out the questionnaire in the presence of the researcher, who intervened as needed. No major difficulties were noted in terms of comprehension of the questions.

\section{Ethics statement}

This study was carried out in respecting ethical rules on the use of human beings. It followed the American Psychological Association's code of conduct for the ethical treatment of human participants. As stated by the Ethical Comity for Non-interventional Research (CERNI - Comite d'Ethique pour les Recherches Non Interventionnelles; IRB00010290 COMUE Université Grenoble-Alpes IRB \#1), in France there is no need for an institutional agreement for simple questionnaire studies (Supplementary Material: Letter on ethics of the CERNI).

\section{Statistical analysis}

\section{Analysis methods}

The data were processed using SPSS 20 software. First, we checked the reliability of the measurement scales using Cronbach's alpha. Reverse statements have been re-coded. Next, we conducted descriptive analyses on the relationships between the variables, using Pearson's correlation coefficient. Then we tested our study hypotheses. Linear regression analyses were computed on the data. For each significant result, we checked to see whether it was moderated by the descriptive (number of training courses and work experience) or sociodemographic (gender, age and education) variables. The moderating effects and any interactions between variables were tested using analyses of variance (ANOVAs). We analysed the interactions to determine whether the link between perceived ability to apply measures and engagement in hygienic and safety behaviours varied as a function of the descriptive and sociodemographic variables. Given that the data for 
Table 1. Descriptive statistics and scale reliability.

\begin{tabular}{lccccccccc}
\hline Variables & $N$ & No. of items & Min & Max & Mean & SD & Med. & Mode & Alpha \\
\hline Fatalistic beliefs & 217 & 6 & 1.00 & 3.50 & 1.94 & 0.44 & 2.00 & 2.00 & 0.68 \\
Perceived risk & 217 & 24 & 1.63 & 4.00 & 2.78 & 0.43 & 2.75 & 2.67 & 0.89 \\
Perceived utility of measures & 217 & 32 & 2.53 & 3.97 & 3.34 & 0.33 & 3.34 & 3.22 & 0.91 \\
Perceived ability (normal periods) & 217 & 32 & 2.56 & 4.00 & 3.41 & 0.35 & 3.43 & 3.25 & 0.90 \\
Perceived ability (rush periods) & 217 & 18 & 1.00 & 4.00 & 2.85 & 0.49 & 2.83 & 2.83 & 0.88 \\
Engagement in hygienic and safety & 217 & 17 & 1.93 & 4.00 & 3.15 & 0.40 & 3.16 & 3.00 & 0.74 \\
$\quad$ & & & & & & & & & \\
\hline
\end{tabular}

all variables were normally distributed (see Table 1), the scores of perceived ability to apply the measures during normal periods and during rush periods were dichotomized at their medians $(\mathrm{Me}=3.43$ and $\mathrm{Me}=2.83$, respectively). Participants whose mean score was below the median were considered to have a low perceived ability to apply the measures, and those whose score was above the median were considered to have a high perceived ability to apply the measures. An analysis of the 2 (perceived ability to apply measures during normal periods)-by-2(gender) interaction was conducted on the data. Finally, in order to find out which of the variables examined had the greatest impact on engagement in hygienic and safety behaviours, we conducted a stepby-step regression analysis. All of the independent variables (perceived ability to apply measures during normal periods and rush periods, fatalistic beliefs, perceived effectiveness of measures and perceived risk) were input as predictors, with engagement in hygienic and safety behaviours as the predicted variable.

\section{Scale reliability test and descriptive analyses}

The measurement scales all obtained very high reliability levels (see Table 1): perceived ability to apply measures during a normal period (32 items, $\alpha=0.90$ ), perceived ability to apply measures during a rush period (18 items, $\alpha=0.88$ ), fatalistic beliefs ( 6 items, $\alpha=0.68$ ), perceived effectiveness of measures (32 items, $\alpha=0.91$ ), perceived risk (24 items, $\alpha=0.89$ ), and engagement in hygienic and safety behaviours (17 items, $\alpha=0.74$ ).

For each scale, the participant's score was obtained by averaging the ratings given for all items on that scale. The higher the participant's score, the more strongly he/she agreed with the statements on the scale. The fatalistic belief scale obtained the lowest mean score $(M=1.94)$. The rest of the scales obtained similar mean scores, which ranged between $M=2.66$ and $M=3.34$. The descriptive analyses indicated that the scores of all variables followed a normal distribution (see Table 1).

\section{Results}

\section{Study population demographics}

The participants ranged in age between 17 and 60 years (mean age $=23$, standard deviation $=5.10$ ), with $55.8 \%$ women and $44.2 \%$ men. Their mean number of years of work experience was 20 months, and over than three-quarters had taken at least one training course in hygiene and safety. Over $90 \%$ of the participants had a high school education or more (see Table 2).

\section{Relationship between study variables}

An analysis of the correlations between the study variables showed that the fatalistic belief scale was negatively correlated with all other scales, which were positively correlated with each other (see Table 3). 
Table 2. Characteristics of the study sample.

\begin{tabular}{|c|c|c|c|c|c|}
\hline Characteristic & $N$ & $\%$ & Characteristic & $N$ & $\%$ \\
\hline \multicolumn{3}{|c|}{ Has attended a training course } & \multicolumn{3}{|l|}{ Gender } \\
\hline Yes & 183 & 84.3 & Male & 96 & 44.2 \\
\hline No & 34 & 15.7 & Female & 121 & 55.8 \\
\hline \multicolumn{3}{|c|}{ No. of training courses attended } & \multicolumn{3}{|l|}{ Age } \\
\hline One & 81 & 44.3 & 20 or younger & 67 & 30.9 \\
\hline Two & 58 & 31.7 & $21-25$ & 109 & 50.2 \\
\hline Three or more & 44 & 24.0 & 26 or older & 40 & 18.4 \\
\hline \multicolumn{3}{|l|}{ Work experience } & \multicolumn{3}{|l|}{ Education } \\
\hline 6 months or less & 76 & 35.0 & Elementary or middle school & 16 & 7.4 \\
\hline $7-12$ months & 41 & 18.9 & High school & 98 & 45.2 \\
\hline $13-24$ months & 54 & 24.9 & Undergraduate studies & 87 & 40.1 \\
\hline 25 months or more & 46 & 21.2 & Graduate studies & 16 & 7.4 \\
\hline
\end{tabular}

Table 3. Correlations between the study variables.

\begin{tabular}{|c|c|c|c|c|c|c|}
\hline Variable & 1 & 2 & 3 & 4 & 5 & 6 \\
\hline 1. Fatalistic beliefs & 1 & & & & & \\
\hline 2. Perceived risk & $-0.168^{*}$ & 1 & & & & \\
\hline 3. Perceived utility of measures & $-0.310^{* *}$ & $0.446^{* *}$ & 1 & & & \\
\hline 4. Perceived ability (normal periods) & $-0.253^{* *}$ & $0.175^{* *}$ & $0.500^{* *}$ & 1 & & \\
\hline 5. Perceived ability (rush periods) & -0.061 & 0.101 & $0.432^{* *}$ & $0.441^{* *}$ & 1 & \\
\hline 6. Engagement in hygienic and safety measures & $-0.269^{* *}$ & $0.176^{* *}$ & $0.423^{* *}$ & $0.381^{* *}$ & $0.379 * *$ & 1 \\
\hline
\end{tabular}
${ }^{*} p<0.05 ;{ }^{* *} p<0.01$.

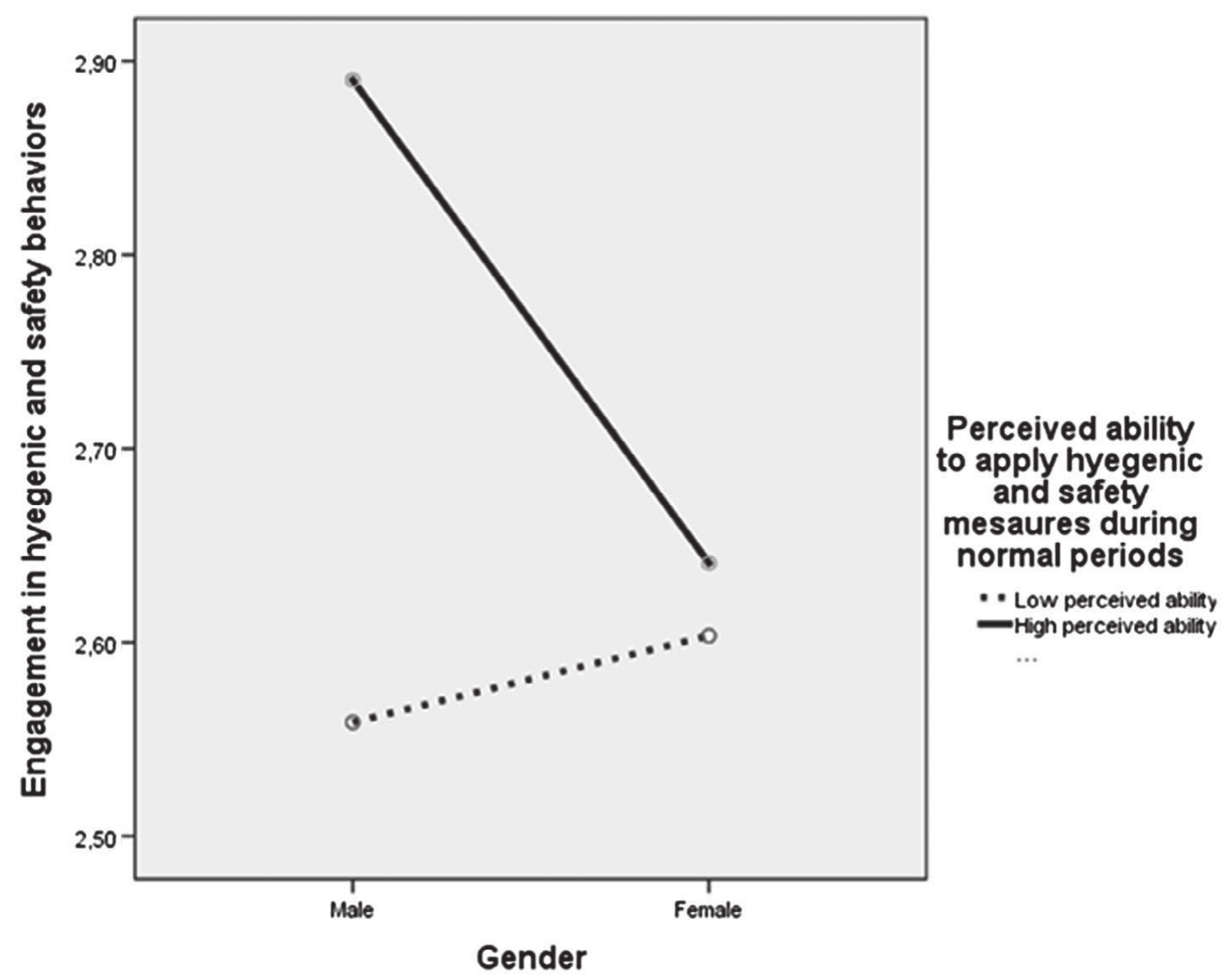

Figure 1. Interaction between gender and perceived ability to apply measures during a normal period. 
Table 4. Effect of work experience on involvement in hygienic and safety behaviours (Tukey's post hoc test).

\begin{tabular}{lccccc}
\hline \multirow{2}{*}{ Experience } & \multicolumn{5}{c}{ Engagement in hygienic and safety behaviours } \\
\cline { 2 - 6 } & $N$ & Mean & Experience & Mean difference & $p$ \\
\hline At most 6 months & 76 & 3.13 & $7-12$ months & 0.03 & 0.983 \\
& & & $13-24$ months & 0.08 & 0.604 \\
7-12 months & 41 & 3.10 & $13-24$ months & 0.05 & 0.888 \\
25 months or more & 46 & 3.31 & At most 6 months & 0.18 & 0.047 \\
& & & 7-12 months & 0.21 & 0.049 \\
& & & $13-24$ months & 0.26 & 0.003 \\
\hline
\end{tabular}

\section{Relationship between perceived ability to apply measures and engagement in hygienic and safety behaviours}

The first analysis indicated that the score on perceived ability to apply hygienic and safety measures during normal periods was a highly significant predictor of the score on engagement in hygienic and safety behaviours, $b=0.43, t(217)=6.04, p<0.001, R^{2}=0.145$. The second analysis showed that the score on perceived ability to apply the measures during rush periods was a highly significant predictor of the score on engagement in hygienic and safety behaviours, $b=0.30, t(217)=6.00, p<0.001$, $R^{2}=0.144$. These two findings validate Hypotheses $1 \mathrm{a}$ and $1 \mathrm{~b}$ : a high perceived ability of employees to apply hygienic and safety measures predicted greater engagement in hygienic and safety behaviours, regardless of the work load.

The main effect of perceived ability to apply measures during normal periods was significant, $F(1$, $213)=26.93, p<0.001, \eta^{2}=0.112$, but the main gender effect was non-significant, $F(1,213)=1.44$, $p=0.231$. By contrast, the interaction between gender and perceived ability to apply measures during normal periods was significant, $F(1,213)=5.58, p=0.019, \eta^{2}=0.026$. Men with a high perceived ability to apply measures during a normal period engaged more in hygienic and safety behaviours than men whose perceived ability to apply those measures was low. This was not the case for women. No interactions were observed during rush periods (Figure 1).

Training had a significant main effect on engagement in hygienic and safety behaviours, $F(1$, $213)=4.52, p=0.035, \eta^{2}=0.021$, as did the number of training courses attended, $F(2,177)=6.58$, $p=0.002, \eta^{2}=0.069$. Participants who had been trained were highly involved in hygienic and safety behaviours $(N=183, M=3.17)$ than did those who had not been trained $(N=34, M=2.99)$. Similarly, participants who had attended several training courses exhibited a greater involvement in hygienic and safety behaviours $(M=3.35)$ than those who had only attended one $(M=3.14, \mathrm{HSD}=0.21, p=0.004)$ or two $(M=3.09$, HSD $=0.25, p=0.002)$ training courses.

The work experience effect was significant, $F(3,209)=4.26, p=0.006, \eta^{2}=0.058$. Participants who had 25 months or over of experience performed more hygienic and safety behaviours than ones who had at most 6 months (HSD $=0.18, p=0.047), 7-12$ months (HSD $=0.21, p=0.049$ ) or 13-24 months ( $\mathrm{HSD}=0.26, p=0.003$ ). The most experienced employees said they carried out more hygienic and safety behaviours than did the less experienced employees (see Table 4). No significant interaction was found between perceived ability to apply measures and any of the other descriptive or sociodemographic variables.

\section{Relationship between fatalistic beliefs and hygienic and safety behaviours}

The link between fatalistic beliefs and engagement in hygienic and safety behaviours was assessed using a simple linear regression analysis, with the fatalistic belief score as the predicting variable and engagement in hygienic and safety behaviours as the predicted variable. Fatalistic beliefs turned out to significantly predict less involvement in hygienic and safety behaviours, $b=-0.24, t(217)=-4.09$, $p<0.001, R^{2}=0.072$. This result validates Hypothesis 2 whereby participants with strong fatalistic beliefs will tend to engage less in preventive behaviours. 
Analyses of the interactions between fatalistic beliefs and the sociodemographic (gender, age and education) and descriptive variables did not result in any significant results.

\section{Relationship between perceived effectiveness of measures and engagement in hygienic and safety behaviours}

Here, we look at the link between the perceived effectiveness of measures and engagement in hygienic and safety behaviours. Perceived effectiveness of hygienic and safety measures was found to predict greater engagement of employees in hygienic and safety behaviours, $b=0.51, t(217)=6.85, p<0.001$, $R^{2}=0.179$. This result supports Hypothesis 3 . Employees were inclined to engage in hygienic and safety behaviours if they felt that the measures they were asked to apply were likely to effectively prevent food contamination.

No significant interactions were observed between the perceived effectiveness of measures and the sociodemographic or descriptive variables.

\section{Relationship between perceived risk and engagement in hygienic and safety behaviours}

The regression analysis revealed that the perceived risk of food contamination significantly predicted engagement in hygienic and safety behaviours, $b=0.16, t(217)=2.62, p=0.009, R^{2}=0.031$. This result is consistent with the Hypothesis 4 which states that participants who perceived that the risk of food contamination was high tended to have a greater engagement in hygienic and safety behaviours.

The interactions with the demographic and descriptive variables were non-significant. Only the interaction between perceived risk and work experience was marginally significant, $F(3,209)=2.53$, $p=0.058, \eta^{2}=0.035$. Participants who perceived the risk of food contamination as high had a greater tendency to engage in hygienic and safety behaviours if they had greater work experience.

\section{Perceived risk as a moderator of the link between self-efficacy beliefs and engagement in hygienic behaviours}

This section examines the interaction between perceived risk and perceived ability to apply measures. A significant main effects of perceived risk, $F(1,213)=6.36, p=0.012, \eta^{2}=0.029$, and perceived ability to apply measures, $F(1,213)=19.88, p<0.001, \eta^{2}=0.085$ was obtained as well as a significant interaction between perceived ability and perceived risk, $F(1,213)=8.22, p=0.005, \eta^{2}=0.037$. Specifically, participants whose perceived ability to apply measures was high engaged in hygienic and safety behaviours if they thought that the risk of food contamination was great. By contrast, those who's perceived ability to apply the measures was low tended to engage less in hygienic and safety behaviours, regardless of whether their perception of the risk was high or low (Figure 2). This finding supports Hypothesis 5 whereby the impact of self-efficacy beliefs on engagement in hygienic and safety behaviours is moderated by the perceived risk of food contamination.

\section{Relative importance of the effects of the variables on engagement in hygienic and safety behaviours}

Finally, it seemed interesting to find out which of the variables examined had the greatest impact on engagement in hygienic and safety behaviours. The results demonstrated that, when all other study variables were controlled, the perceived effectiveness of the measures turned out to be the variable that explained the greatest amount of engagement in hygienic and safety behaviours $\left(R^{2}=0.179\right)$. Perceived ability to apply the measures during a rush was the second explanatory variable of engagement in hygienic and safety behaviours: it increased the percentage of explained variance by $4.7 \%$ $\left(\Delta R^{2}=0.047\right)$. The perceived effectiveness of the measures and the perceived ability to apply them during a rush period accounted for $22.6 \%$ of the variance in hygienic- and safety-behaviour involvement 


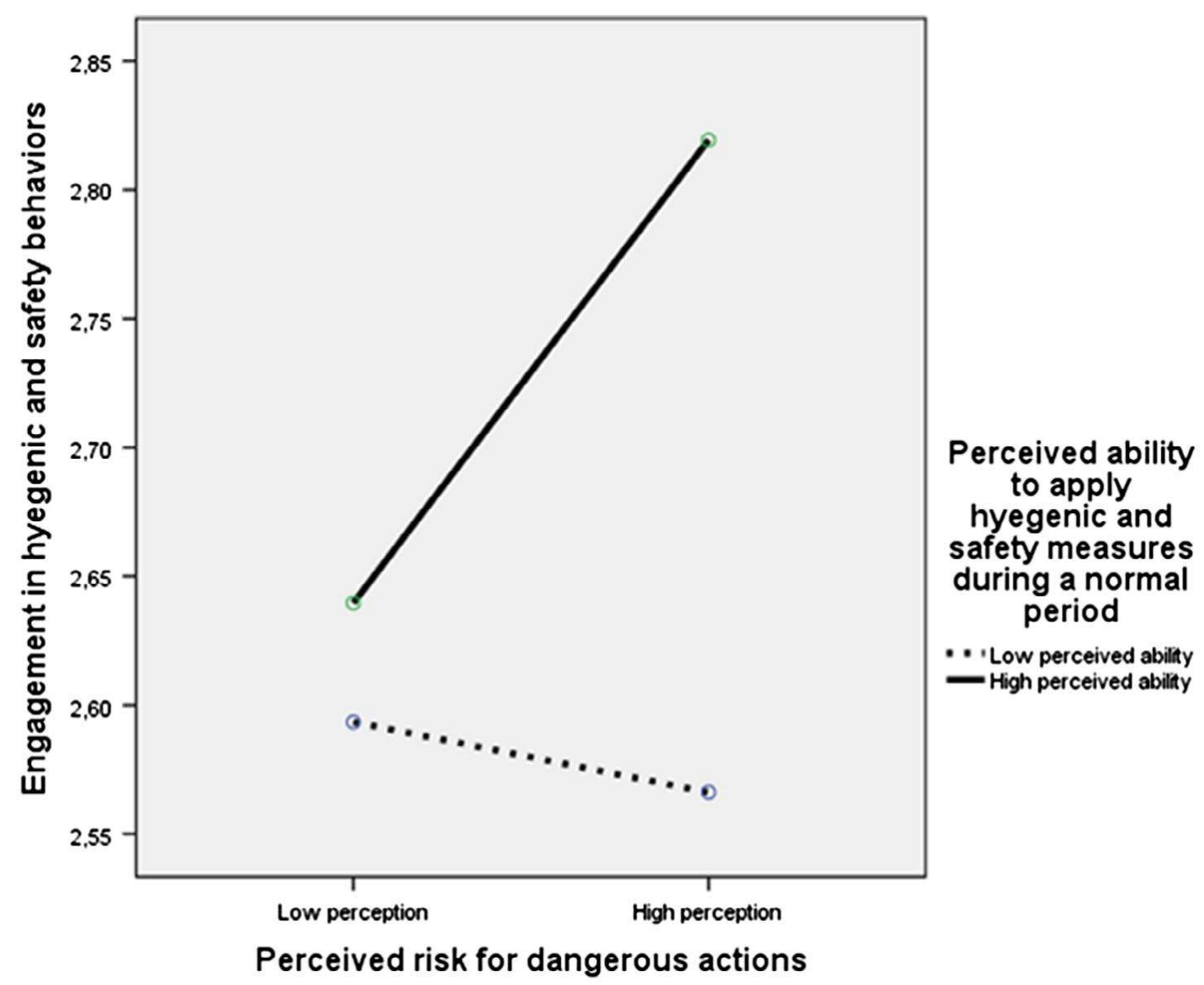

Figure 2. Interaction between perceived ability to apply measures and perceived risk.

Table 5. Summary of step-by-step regression analyses on the study variables.

\begin{tabular}{lcccccc}
\hline & & \multicolumn{4}{c}{ Dependent variable: engagement in hygienic and safety } \\
behaviours \\
\cline { 3 - 7 } Model & Predicting variable & $b$ & $t$ & $p$ & $R^{2}$ & $\Delta R^{2}$ \\
\hline 1 & Perceived utility of measures & 0.51 & 6.85 & $<0.001$ & 0.179 & 0.179 \\
2 & Perceived ability during rush periods & 0.19 & 3.61 & $<0.001$ & 0.226 & 0.047 \\
3 & Fatalistic beliefs & -0.16 & -2.77 & 0.006 & 0.253 & 0.027 \\
\hline
\end{tabular}

$\left(R^{2}=0.179+0.047=0.226\right)$. Fatalistic beliefs were the third variable contributing to engagement in hygienic and safety behaviours. It increased the explained variance by $2.7 \%\left(\Delta R^{2}=0.027\right)$ (see Table 5). Perceived risk no longer explained a significant part of the variance in hygienic- and safety-behaviour execution when the other variables were controlled.

\section{Discussion}

The findings of this study are consistent with our hypotheses. Employees' perceived ability to apply hygienic and safety measures was indeed a predictor of their greater involvement in hygienic and safety behaviours, regardless of whether the work load was normal or heavy. This result aligns with earlier studies showing that self-efficacy is a key variable in the adoption of protective behaviours (Strecher et al. 1986; Schwarzer 1992; Bandura 1997; Luszcynska 2004; Phillip \& Anita 2010; Van Zundert et al. 2010; Milton \& Mullan 2012). Clearly, perceptions of one's ability to implement an action seem to be decisive in generating the intention to implement and execute that action. This perceived ability "stimulates confidence, effort, and the will to persevere in carrying out the action, even in the presence 
of obstacles" (Kouabenan 2006, p. 271). It follows, then, that employees who feel capable of implementing hygienic and safety measures are the ones who report doing so to the greatest extent. This disposition seems to be reinforced by both training and work experience. Accordingly, we found that participants who had attended several training courses in hygiene and safety and those with the most experience said they performed hygienic and safety behaviours.

It follows that fatalistic beliefs, which reflect a lack of control, gave rise to the opposite result, that is, individuals who had strong fatalistic beliefs reported less involvement in hygienic and safety behaviours. This second finding confirms those of earlier studies showing that fatalistic beliefs can have a negative effect on execution of safety-related actions, and can lower motivation to engage in safety-related behaviours (Kouabenan 1998; Peltzer \& Renner 2003; Claassen et al. 2010; Kayani et al. 2012). In Kouabenan's (1998) study fatalists generally tended to ascribe road accidents to factors over which the driver had no control and to minimise the role of factors involving initiatives on their part. For this author, "fatalistic beliefs, which can lead to a low sense of control over events, are likely to elicit resignation and passiveness with respect to safety and self-protective measures" (Kouabenan 2001, p. 339). For Claassen et al. (2010), a fatalistic belief "is a belief that little can be done to change the risk", and can "adversely affect motivation to engage in risk-reducing behaviour" (p. 184).

Thirdly employees had a greater tendency to engage in hygienic and safety behaviours when they believed those behaviours were likely to prevent food contamination. This result underlines the importance of believing in the perceived effectiveness of a response or of a safety measure in motivation to take action. The fact of believing there exists an appropriate behaviour for coping with a threat is claimed by certain authors, and rightly so, to be a critical element in precaution-taking processes (Rogers 1983; Becker \& Rosenstock 1987; Weinstein 1993; Schwarzer 1994; Dejoy 1996). For Becker and Rosenstock (1987), having the conviction that abiding by a particular recommendation for prevention is an effective way of reducing the perceived threat (in terms of its costs - obstacles, efforts) to a level that is subjectively acceptable. Indeed, to apply a protective measure, one must believe in the ability of a given action or behaviour to lower the probability of occurrence of the adverse event or reduce its consequences (Weinstein 1993).

Another important finding of the present study is that participants who perceived a high risk of food contamination had a tendency to perform hygienic and safety behaviours. This finding confirms the results of many studies demonstrating the impact of perceived risk on the execution of precautionary behaviours (Dejoy 1996; Kuttschreuter 2006; Weinstein et al. 2007; Arezes \& Miguel 2008; Kouabenan 2009; Mbaye \& Kouabenan 2013). In this direction, Kuttschreuter (2006) found that persons who said they would avoid eating allegedly contaminated chicken perceived the risk as higher, were more worried, saw themselves as very vulnerable, needed large amounts of information and were less confident about the safeness of food. Moreover, we found an interesting interaction between perceived ability and perceived risk indicating that perceived risk moderated the impact of self-efficacy beliefs on engagement in hygienic and safety behaviours: participants who had a high perceived ability to apply the measures during a normal work period reported engaging in hygienic and safety behaviours when they perceived the risk as great.

A final innovative result of this study is that the perceived effectiveness of preventive measures was the variable that best explained employee involvement in hygienic and safety behaviours. Self-efficacy was the second explanatory variable. To initiate and maintain hygienic and safety behaviours, one must first believe in their effectiveness, and then believe in one's personal ability to implement them. Although various authors have stressed the importance of the perceived effectiveness of preventive measures (Rogers 1983; Becker \& Rosenstock 1987; Weinstein 1988; Schwarzer 1994; Dejoy 1996), we know of no studies showing that this factor carries more weight than perceived self-efficacy. Perceived self-efficacy has often been said to be the most influential factor in determining safety-related behaviour (Schwarzer 1992; Bandura 1997). 


\section{Limitations and direction for future research}

It is important to note several limitations of this study. The first is that we studied self-reported behaviours, not observed behaviours. Even though we anticipated this bias in the instructions by explicitly asking the participants to base their responses on what they actually do at work, we can still raise the question of the social desirability of the employees' responses. Employees may have been tempted to respond in line with rules and regulations rather than in terms of what they really do. Note, however, that Milton and Mullan (2012) found a very strong positive link between self-reported behaviours and real behaviours. Furthermore, this bias may have been reduced by the collecting of the data in a face-to-face interview and our presence in the restaurants for several days during the study. Another limitation of the study is that we conducted correlation analyses, which means that we cannot draw any conclusions regarding causality. It is difficult to conclude with certainty whether it was perceived self-efficacy that affected observance of hygienic and safety measures or the fact that applying recommended measures increases self-efficacy. Further studies are necessary to take into account these limitations. For example, it would be interesting to see if as Milton and Mullan (2012), we obtain similar results for actual behaviour and reported behaviour. Similarly, since few significant results were found for the descriptive (number of training courses, work experience) or sociodemographic (gender, age and education) variables, it would be interesting to test in a future study simple and interaction effects of these variables on engagement in hygienic and safety behaviours as well as on perceived ability to apply measures during normal periods and rush periods, fatalistic beliefs and perceived effectiveness of prevention measures.

\section{Practical implications and conclusions}

Despite these limitations, the present study offers some interesting results in the area of prevention. It shows that awareness of hygiene-related measures is necessary but insufficient to trigger hygiene-related behaviours (Clayton et al. 2002). It suggests the effectiveness of designing targeted preventive actions that take into account the beliefs of the target population. It would seem important to design actions and messages aimed at generating beliefs not only in the effectiveness of preventive measures but also in the ability of workers to implement them. This could be done both by stressing the effectiveness of hygienic and safety measures and by creating conditions for their implementation that are less effortful, are compatible with production requirements (inclusion in work hours), and are valorised within the company. Training programs or company actions should demonstrate employees of the effectiveness of preventive measures rather than simply informing them of safety measures. At the same time, they should enhance each employee's perceived ability to mobilise his/her resources and engage in actions aimed at preventing the risks of food contamination. One can increase this self-efficacy belief by taking the naive perceptions of workers into account and allowing them to take part in designing safety measures (Kouabenan 2006, 2009). When workers are allowed to participate, they will not only feel that they "count" but will also understand the usefulness of the measures and be more motivated to apply them, even if the suggestions and problems they bring up are not always taken into consideration. The sharing of experiences during training could help reveal the inconsistencies and difficulties employees encounter in implementing hygienic and safety measures (time constraints, lack of staff and lack of resources) and could thereby increase perceived efficacy. This requires an open safety climate that supports and values safety-promoting actions and initiatives, encourages employees to make hygiene- and safety-related requests, and establishes a climate of trust (see Kouabenan et al. 2015).

\section{Disclosure statement}

No potential conflict of interest was reported by the authors. 


\section{Funding}

This study was funded by L'Institut Quick.

\section{References}

Ajzen I. 1985. From intentions to actions: a theory of planned behaviour. In: Kuhl J, Beckmann J, editors. Action control. Heidelberg: Springer; p. 11-39.

Albarracín D, Johnson BT, Fishbein M, Muellerleile PA. 2001. Theories of reasoned action and planned behavior as models of condom use: a meta-analysis. Psychol Bull. 127:142-161. doi:10.1146/annurev.psych.52.1.27.

Arezes PM, Miguel AS. 2008. Risk perception and safety behaviour: a study in an occupational environment. Safety Sci. 46:900-907. doi:10.1016/j.ssci.2007.11.008.

Armitage CJ, Conner M. 1999. The theory of planned behaviour: assessment of predictive validity and perceived control. Br J Soc Psychol. 38:35-54.

Bandura A. 1977. Self-efficacy: toward a unifying theory of behavioral change. Psychol Rev. 84:191-215.

Bandura A. 1997. Self-efficacy: the exercise of control. New York, NY: Freeman.

Becker MH, Rosenstock IM. 1987. Comparing social learning theory and the health belief model. In: Ward WB, editor. Advances in health education and promotion (vol. 2). Greenwich: JAI Press; p. 245-249.

Bergvik S, Sørlie T, Wynn R. 2012. Coronary patients who returned to work had stronger internal locus of control beliefs than those who did not return to work. Br J Health Psychol. 17:596-608. doi:10.1111/j.2044-8287.2011.02058.x.

Brewer NT, Chapman GB, Gibbons FX, Gerrard M, McCaul KD, Weinstein, ND. 2007. Meta-analysis of the relationship between risk perception and health behavior: the example of vaccination. Health Psychol. 26:136-145.

Chow S, Mullan B. 2010. Predicting food hygiene. An investigation of social factors and past behaviour in an extended model of the health action process approach. Appetite. 54:126-133. doi:10.1016/j.appet.2009.09.018.

Claassen L, Henneman L, De Vet R, Knol D, Marteau T, Timmermans D. 2010. Fatalistic responses to different types of genetic risk information: exploring the role of self-malleability. Psychol Health. 25:183-196.

Clayton DA, Griffith CJ, Price P, Peters AC. 2002. Food handlers' beliefs and self-reported practices. Int J Environ Health Res. 12:25-39.

Clayton DA, Griffith CJ. 2008. Efficacy of an extended theory of planned behaviour model for predicting caterers' hand hygiene practices. Int J Environ Health Res. 18:83-98. doi:10.1080/09603120701358424.

de Zwart O, Veldhuijzen IK, Elam G, Aro AR, Abraham T, Bishop GD, Voeten HACM, Richardus JH, Brug J. 2009. Perceived threat, risk perception, and efficacy beliefs related to SARS and other (emerging) infectious diseases: results of an international survey. Int J Behav Med. 16:30-40.

Dejoy DM. 1996. Theoretical model of health behaviour and work-place self-protective behaviour. J Safety Res. 27:62-72. doi:10.1016/0022-4375(96)00007-2.

Egan MB, Raats MM, Grubb SM, Eves A, Lumbers ML, Dean MS, Adams MR. 2007. A review of food safety and food hygiene training studies in the commercial sector. Food Control. 18:1180-1190. doi:10.1016/j.foodcont.2006.08.001.

Graffeo M, Savadori L, Tentori K, Bonini N, Rumiati R. 2009. Consumer decision in the context of a food hazard: the effect of commitment. Mind Soc. 8:59-76.

Griffith CJ, Livesey KM, Clayton D. 2010. The assessment of food safety culture Br Food J. 112:439-456.

Houghton JR, van Kleef EV, Rowe G, Frewer LJ. 2006. Consumer perceptions of the effectiveness of food risk management practices: a cross-cultural study. Health Risk Soc. 8:165-183. doi:10.1080/13698570600677373.

Kayani A, King MJ, Fleiter JJ. 2012. Fatalism and its implications for risky road use and receptiveness to safety messages: a qualitative investigation in Pakistan. Health Educ Res. 27:1043-1054. doi:101093/her/cys096.

Kouabenan DR. 1998. Beliefs and the perception of risks and accidents. Risk Anal. 18:243-252. doi:10.1111/j.1539-6924.1998. tb01291.x.

Kouabenan DR. 2001. Culture, perception des risques et explication des accidents [Culture, risk perception and accidents' explanation]. Bull Psychol. 54:327-342.

Kouabenan DR. 2006. From beliefs to protective behaviours - 2nd part: What contributions of the studies on risk perception in the diagnosis of safety and in the prevention initiatives? In: D.R. Kouabenan, B. Cadet, D. Hermand, M.T. Muñoz Sastre (Eds). Psychology of risk: Identify, assess and prevent. Brussels: De Boeck; p. 259-289.

Kouabenan DR. 2007. Incertitude, croyances et management de la sécurité [Uncertainty, beliefs, and safety management]. Le travail humain. 70:271-287.

Kouabenan DR. 2009. Role of beliefs in accident and risk analysis and prevention. Safety Sci. 47:767-776. doi:10.1016/j. ssci.2008.01.010.

Kouabenan DR, Ngueutsa R, Mbaye S. 2015. Safety climate, perceived risk, and involvement in safety management. Safety Sci. 77:72-79.

Kuttschreuter M. 2006. Psychological determinants of reactions to food risk messages. Risk Anal. 26:1045-1057. doi:10.1111/j.1539-6924.2006.00799.x. 
Luszczynska A. 2004. Change in breast self-examination behavior: effects of intervention on enhancing self-efficacy. Int J Behav Med. 11:95-103.

Mbaye S, Kouabenan DR. 2013. Effects of the feeling of invulnerability and the feeling of control on motivation to participate in experience-based analysis, by type of risk. Accid Anal Prev. 51:310-317. doi:10.1016/j.aap.2012.11.026.

McCaul KD, Sandgren AK, O’Neill HK, Hinsz VB. 1993. The value of the theory of planned behavior, perceived control, and self-efficacy expectations for predicting health-protective behaviors. Basic Appl Soc Psychol. 14:231-252.

Milton AC, Mullan BA. 2010. Consumer food safety education for the domestic environment: a systematic review. Br Food J. 112:1003-1022.

Milton AC, Mullan BA. 2012. An application of the theory of planned behavior - a randomized controlled food safety pilot intervention for young adults. Health Psychol. 31:250-259.

Mullan BA, Wong C. 2009. Hygienic food handling behaviours. An application of the theory of planned behaviour. Appetite. 52:757-761.

National Institute for Public Health Surveillance - InVS 2011. Morbidity and mortality from foodborne infectious disease in France. Retrived from http://www.invs.sante.fr/publications/2004/inf_origine_alimentaire/inf_origine_ alimentaire.pdf

Powell D, Jacob C, Chapman B. 2011. Enhancing food safety culture to reduce rates of foodborne illness. Food Control. 22:817-822.

Peltzer K, Renner W. 2003. Superstition, risk-taking and risk perception of accidents among South African taxi drivers. Accid Anal Prev. 35:619-623.

Phillip S, Anita E. 2010. Efficacy of the theory of planned behaviour model in predicting safe food handling practices. Food Control. 21:983-987.

Riley GA, Baah-Odoom D. 2012. Belief in a just world, generalised self-efficacy and stigma may contribute to unsafe sexual intentions via a reduced perception of vulnerability to HIV/AIDS amongst young people in Ghana. AIDS Care. 24:642-648.

Rimal RN, Real K. 2003. Perceived risk and s beliefs as motivators of change. Use of the risk perception attitude (RPA) framework to understand health behaviours. Hum Commun Res. 29:370-399.

Rogers RW. 1983. Cognitive and physiological processes in fear appeals and attitude change. A revisited theory of protection motivation. In: Cacioppo J, Petty RE, editors. Social psychophysiology. New York, NY: Guilford Press; p. 153-176.

Schwarzer R. 1992. Self-efficacy in the adoption and maintenance of health behaviours: theoretical approaches and a new model. In: Schwarzer R, editor. Self-efficacy: thought control of action. Washington, DC: Hemisphere; p. $217-242$.

Schwarzer R. 1994. Optimism, vulnerability, and self-beliefs as health-related cognitions: a systematic overview. Psychol Health. 9:161-180.

Sloan FA, Paron NA, Platt AC. 2009. Preferences, beliefs, and self-management of diabetes. Health Res Educ Trust. 44:1068-1087.

Slovic P, Fischhoff B, Lichtenstein S. 1981. Perceived risk: psychological factors and social implications [and discussion]. Proc R Soc A Math Phys Eng Sci. 376:17-34. doi:10.1098/rspa.1981.0073.

Stasson M, Fishbein M. 1990. The relation between perceived risk and preventive action: a within-subject analysis of perceived driving risk and intentions to wear seatbelts. J Appl Soc Psychol. 20:1541-1557. doi:10.1111/j.1559-1816.1990. tb01492.x.

Strecher VJ, DeVellis BM, Becker MH, Rosenstock IM. 1986. The role of self-efficacy in achieving health behavior change. Health Educ Behav. 13:73-92.

van der Pligt J. 1996. Risk perception and self-protective behavior. Eur Psychol. 1:34-43. doi:10.1027/1016-9040.1.1.34.

Van Zundert RMP, Ferguson SG, Shiffman S, Engels RCME. 2010. Dynamic effects of self-efficacy on smoking lapses and relapse among adolescents. Health Psychol. 29:246-254.

Vaughan E. 1993. Chronic exposure to an environmental hazard: risk perceptions and self-protective behavior. Health Psychol. 12:74-85.

Weinstein ND. 1988. The precaution adoption process. Health Psychol. 7:355-386.

Weinstein ND. 1993. Testing four competing theories of health-protective behavior. Health Psychol. 12:324-333.

Weinstein ND, Kwitel A, McCaul KD, Magnan RE, Gerrard M, Gibbons FX. 2007. Risk perceptions: assessment and relationship to influenza vaccination. Health Psychol. 26:146-151. doi:10.1037/0278-6133.26.2.146.

Wright M, Leach P. 2013. Diagnosing and improving food safety culture in food businesses. Available from https://www. aibonline.org/press/AIBStatement04022009/PCASituation.html

Yiannas F. 2009. Food safety culture: creating a behavior-based food safety management system (food microbiology and food safety). New York, NY: Springer Science. doi:10.1007/978-0-387-72867-4. 